Abstract IDDF2021-ABS-0127 Table 1 Association of Helicobacter Pylori with obesity in the western Population of kingdom of Suadi Arabia

\begin{tabular}{lll}
\hline BMI & HP Positive (\%) & HP Negative (\%) \\
\hline $30-34$ & 32 & 68 \\
$35-39$ & 29 & 71 \\
$40-44$ & 23 & 77 \\
$50-54$ & 54 & 55 \\
$55-59$ & 50 & 50 \\
$60-64$ & 60 & 40 \\
$>65$ & 73 & 27 \\
\hline
\end{tabular}

hospital information system and their demographics, comorbidities and HP status were recorded retrospective.

Results 468 obese patients (male: female, 1:1.7) with mean age of 48.3 (SD \pm 15 ) years and mean BMI of $44.43 \mathrm{~kg} /$ $\mathrm{m}^{2}$ were analyzed. The prevalence of $\mathrm{HP}$ was $35 \%$ (166/ 469). There was no significant association of HP infection and gender $(\mathrm{P}=0.557)$. There was no difference between the mean ages of patients infected with HP $(40.2 \pm 12$ years) compared to those who were not (42.46 \pm 13.7 years) $(P=0.076)$. Similarly was the case with glycated haemoglobin $(6.79 \pm 1.9$ in HP positive compared with $6.85 \pm 1.7$ in HP negative, $\mathrm{P}=0.708)$. However, the mean BMI was significantly higher in $\mathrm{HP}$ positive patients $\left(46.75 \pm 10.3 \mathrm{~kg} / \mathrm{m}^{2}\right)$ compared to $\mathrm{HP}$ negative (43.1 \pm $\left.8.2 \mathrm{~kg} / \mathrm{m}^{2}\right)(\mathrm{P}=0.0001)$. There was a positive linear correlation with $\mathrm{BMI}$ and $\mathrm{HP}$ prevalence $(\mathrm{P}=0.0007)$ (IDDF2021-ABS-0127 Table 1).

Conclusions The prevalence of HP in our study was positively associated with BMI, rising with increasing BMI. This may have implications for obesity management. Further populationbased studies are needed to confirm.

\section{IDDF2021-ABS-0128 SELF-EXPANDING METALLIC STENT FOR PALLIATION OF MALIGNANT COLORECTAL CANCER: A SINGLE TERTIARY REFERRAL CENTER EXPERIENCE}

${ }^{1}$ Adnan Alzanbagi* ${ }^{1}$ laegue Ahmed, ${ }^{2}$ Mohammed Fatani, ${ }^{2}$ Hussan Alaidarous, IIshtiaque Ahmed, 'Abdulaziz Tashkandi, ${ }^{1}$ Mohammed Khan, ${ }^{1}$ Ali Algesry, ${ }^{1}$ Mohammed Shariff. 'King Abdullah Medical City Makkah, Saudi Arabia; ${ }^{2}$ Umm Al Qura University Makkah, Saudi Arabia

10.1136/gutjnl-2021-IDDF.149

Background 13\% of CRC in the late stage present with intestinal obstruction and there is no clear consensus on how best to manage this clinical emergency. The purpose of this study was to evaluate the effectiveness of Self-expanding metallic stents (SEMS) placement in CRC stenoses in the western region of KSA.

Methods A retrospective review of SEMS placed in King Abdullah Medical City, Makkah, for histological proven inoperable CRC stenosis was done including, demographics data, the success of stent placement, complication, re-intervention and mortality.

Results 26 SEMS were placed in 25 patients. Median age was 51 years (range 37-84) with 64\% (16) males, all CRC had distant metastasis and involved the left side of the colon, except for one located in the hepatic flexure. All SEMS were uncovered with mean length $9 \mathrm{~cm}$. SEMS was placed successfully in all the cases, except one (96\%). Two SEMS placements in sigmoid resulted in perforation requiring emergency surgery, both survived till date. One of the SEMS failed to expand despite appropriate placement ending in colostomy. Tumor in-growth in one case resulted in further obstruction 6 months following SEMS placement. This was successfully treated by restenting with SEMS. There was no SEMS migration or bleeding. Mortality was 52\% (13). Chemotherapy was given to $56 \%$ (14) with one having a perforation. Clinical success of relieving obstruction without complication was achieved in $88 \%$.

Conclusions Placement of SEMS for palliation of obstruction due to CRC in our center was effective in relieving colonic obstruction with complication rates comparable to other studies.

\section{IDDF2021-ABS-0129 METALLIC STENT INSERTION FOR PALLIATION OF ESOPHAGEAL CANCER: SINGLE-CENTRE EXPERIENCE FROM SAUDI ARABIA}

${ }^{1}$ Laeeque Ahmed*, ${ }^{1}$ Adnan Alzanbagi, ${ }^{1}$ Ishtiaque Ahmed, ${ }^{2}$ Bashaer Alzahrani, ${ }^{2}$ Hosam Alghanmi, ${ }^{1}$ Mohammed Shariff. ${ }^{1}$ King Abdullah Medical City Makkah, Saudi Arabia; ${ }^{2}$ Umm Al Qura University Makkah, Saudi Arabia

\subsection{6/gutjpl-2021-IDDF.150}

Background The use of Self-Expandable metallic stents (SEMS) as a non-surgical alternative for palliation of advanced esophageal cancer (EC) is increasing. However, there is a scarcity of real-life experience on the use of these stents exclusively in oesophageal cancer. The aim of this study is to evaluate the efficacy of SEMS in inoperable esophageal cancers in the western region of Saudi Arabia.

Methods A retrospective review of SEMS placed in a tertiary referral hospital for histological proven inoperable EC from 2012 until 2015. Demographics data, procedure success, complication, re-intervention and mortality were analyzed.

Results 46 SEMS were placed in 35 patients for palliation of dysphagia. Median age of patients was 68 years (range 31. 95). 69\% (24) patients have lower third of EC and the rest have middle third (IDDF2021-ABS-0129 Table 1). SEMS was placed successfully in all cases with symptomatic improvement. No major stent-related complication seen. $28 \%$ (13) patients required re-intervention with additional SEMS placement, 9 of which were for tissue in growth and 4 for distal migration. Median survival was 114 days (range 30 - 498). Most of the complications seen in fully covered SEMS compared to the partially covered $50 \%(8 / 16)$ vs $17 \%(5 / 30)$,

Abstract IDDF2021-ABS-0129 Table 1 Characteristics of patients who received SEMS

\begin{tabular}{ll}
\hline Characteristics & $\begin{array}{l}\text { Number }=35 \\
\text { (percentage) }\end{array}$ \\
\hline Age: median (range) & $68(31-95)$ \\
Gender: male: female & $0.8: 1$ \\
Oesophageal Cancer Location Upper Middle Lower & $0(0) 11(31 \%) 24(69 \%)$ \\
Oesophageal cancer type Adenocarcinoma Squamous & $27(77 \%) 8(23 \%)$ \\
carcinoma & $18(51 \%) 17(49 \%)$ \\
Received Chemo/Radiotherapy Yes No & \\
\hline
\end{tabular}




\begin{tabular}{ll} 
Abstract IDDF2021-ABS-0129 Table 2 & Complications \\
\hline Complications & Patients, $\mathbf{n}(\%)$ \\
\hline Perforation & $0(0 \%)$ \\
Bleeding & $0(0 \%)$ \\
Tumor in/over growth & $9(20 \%)$ \\
Stent migration & $4(9 \%)$ \\
Death & $0(0 \%)$ \\
\hline
\end{tabular}

respectively $\mathrm{p}=0.04$ (IDDF2021-ABS-0129 Table 2). Chemo and/or radiotherapy were given to $51 \%$ (18) of the patients without any significant benefit on survival $(p=0.79)$ or reintervention rate $(\mathrm{p}=0.47)$ compared to those who did not.

Conclusions SEMS is effective in palliating dysphagia in inoperable EC without major complications. Rates of tumor ingrowth and migration were comparable to other studies. SEMS provides long-term palliation.

\section{IDDF2021-ABS-0131 MIXED TYPE EARLY GASTRIC CANCER SHOWS MORE AGGRESSIVE BEHAVIOR: A NETWORK META-ANALYSIS}

Peng Yang ${ }^{*}$ XiaoYong Wang. The Affiliated Changzhou No. 2 People's Hospital of Nanjing Medical University, China

\subsection{6/gutjnl-2021-IDDF.151}

Background To date, the biological behavior about EGC (early gastric cancer) with pure differentiated type (PD), pure undifferentiated type (PU) and MD (Differentiated/Undifferentiated Mixed Type) still remains controversy. Thus, this study aimed to compare the biological behavior among PD, PU and MT through a network meta-analysis.

Methods Studies from inception to April 2021 were searched using the PubMed, Web of Science and Embase databases. The odds ratios (OR) was used to combine the effect sizes, and the interval estimation was performed with $95 \%$ confidence intervals (CIs). Inconsistency factor and 95\% CIs were used to evaluate the consistency of each closed loop. Surface under the cumulative ranking curve (SUCRA) was used to rank the results of the network metaanalysis. Lastly, publication bias was assessed by creating the funnel plots. All statistical analyses were performed by Stata software, version 15.1.

Results Our study included a total of 14 articles involving 12239 patients with early gastric cancer. Network meta-analysis revealed that Patients with MT EGC had significantly higher risks of lymph node metastasis (LNM) (MT vs PU: OR, 1.92; 95\% CI, 1.48-2.49. MT vs PD: OR, 3.68; 95\% CI, 2.78-4.87)(IDDF2021-ABS-0131 Figure 1. Comparison of the risks of lymph node metastasis among PD, PU and MT EGC), submucosal invasion (MT vs PU: OR, 2.06; 95\% CI, 1.27-3.33. MT vs PD: OR, 1.47; 95\% CI, 0.91-2.36) (IDDF2021-ABS-0131 Figure 2. Comparison of the risks of submucosal invasion among PD, PU and MT EGC), lymphovascular invasion (MT vs PU: OR, 1.91; 95\% CI, 1.13-3.23. MT vs PD: OR, 1.82; 95\% CI, 1.11-2.99) (IDDF2021-ABS0131 Figure 3. Comparison of the risks of lymphovascular invasion among PD, PU and MT EGC), and larger size (tumor size2 cm) (MT vs PU: OR, 1.46; 95\% CI, 1.18-1.80. MT vs PD: OR, 1.87; 95\% CI, 1.55-2.26) (IDDF2021-ABS-0131 Figure 4. Comparison of the risks of larger size (tumor size $>2$ $\mathrm{cm}$ ) among PD, PU and MT EGC) compared with PD and PU EGC patients.

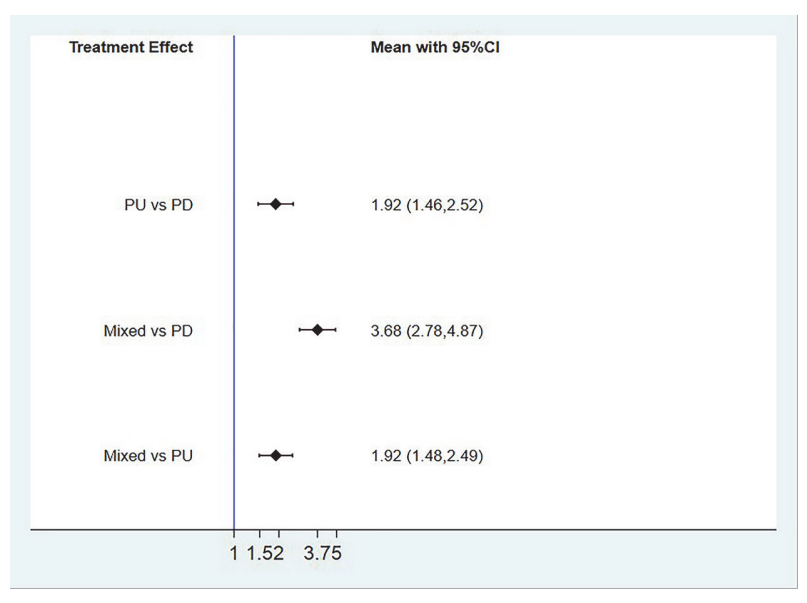

Abstract IDDF2021-ABS-0131 Figure 1

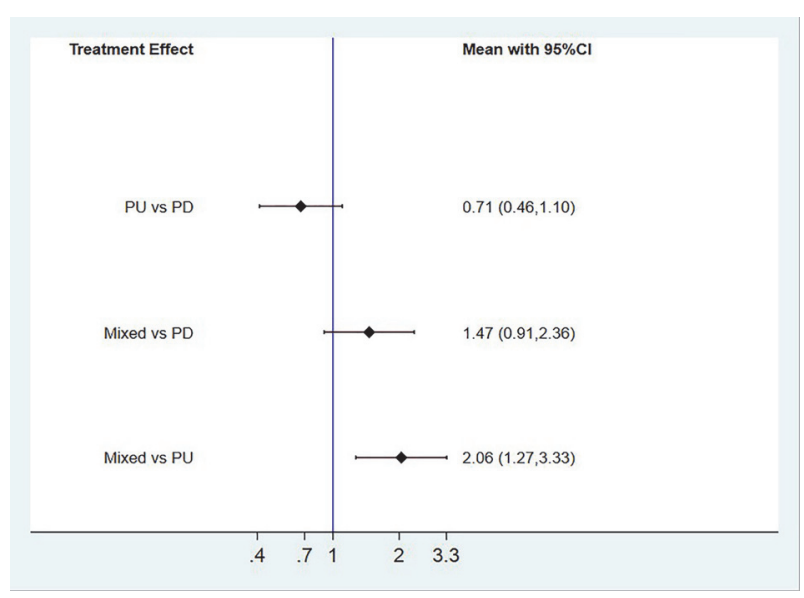

Abstract IDDF2021-ABS-0131 Figure 2

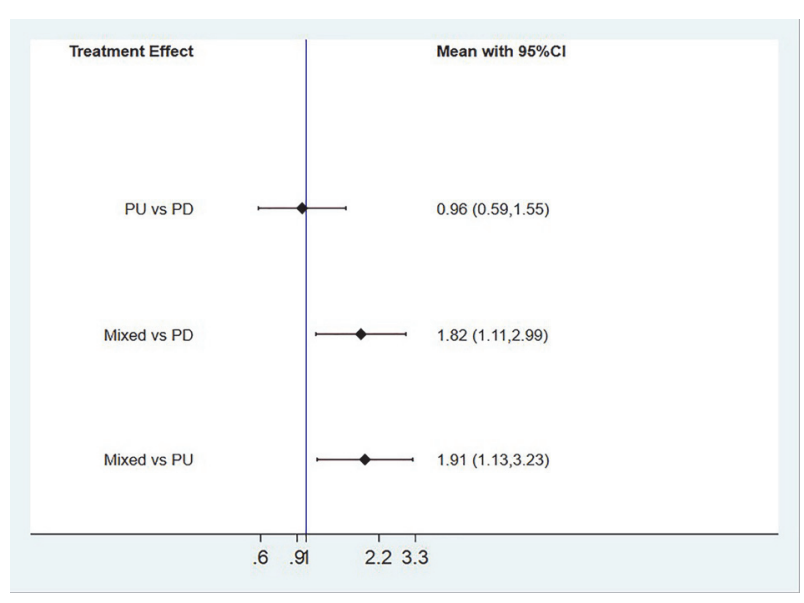

Abstract IDDF2021-ABS-0131 Figure 3 